\title{
Lesions of the optic radiations mimicking lateral geniculate nucleus visual field defects
}

\author{
JOHN E CARTER, ${ }^{*}$ PATRICK O’CONNOR, $†$ DAVID SHACKLETT, $\dagger$ \\ MICHAEL ROSENBERG, $\ddagger$
}

\begin{abstract}
From the Departments of Medicine (Neurology)* and Ophthalmology, $\dagger$ University of Texas Health Science Center, San Antonio, and Department of Neurology, $\ddagger$ Uniformed Services, University for the Health Sciences, Bethesda, Maryland, USA
\end{abstract}

SUMMARY Three patients were found to have visual field defects which involved homonymous horizontal sectors of the visual fields, thus mimicking field defects associated with lesions of the lateral geniculate nucleus. Computed tomography provided anatomical evidence that the responsible lesion involved the optic radiations rather than the lateral geniculate nucleus.

Visual field defects involving horizontal sectors of the homonymous visual fields are rare. They do not even appear in reports discussing large series of patients with homonymous hemianopic visual field defects. ${ }^{12}$ Nevertheless, a homonymous horizontal sectoranopia was described as early as $1931 .^{3}$ Earlier authors considered homonymous horizontal sectoranopia an indication of a lesion in the visual radiations. ${ }^{45}$ More recent authors have argued that a lesion of the lateral geniculate nucleus is more likely..$^{6-10}$ Indeed, Hoyt states that earlier cases of homonymous horizontal sectoranopia were caused by unrecognised lateral geniculate nucleus lesions. ${ }^{8}$ The present report provides anatomical evidence from computed tomography that homonymous horizontal sectoranopias may be associated with lesions situated in the cerebral hemispheres. By their location, these lesions must primarily invade or compress the optic radiations rather than the lateral geniculate nucleus.

\section{Case reports}

Case 1. A 26-year-old right-handed black male was brought to the hospital because of an episode of loss of consciousness. He had been travelling cross-country by bus and had had little food intake for 24-48 hours. He had been drinking alcohol in uncertain quantities when he had a generalised convulsion on the bus. He had recently been discharged from military service and denied past history of

Address for reprint requests: John E Carter, MD, Department of Medicine (Neurology), University of Texas Health Science Center, San Antonio, Texas 78284, USA

Received 15 February 1985. Accepted 23 March 1985. seizures or other significant medical problems. Examination was entirely unremarkable except for an initial period of mild drowsiness on arrival to the hospital. No lateralising findings were present and on confrontation visual field $\stackrel{\oplus}{.}$ testing no field defect was found. On formal perimetry $\overrightarrow{0}$ left homonymous horizontal sectoranopia was demons ${ }^{\circ}$ trated (fig 1). Routine and sleep-deprived electroenci $\stackrel{\infty}{N}$ phalograms (EEGs) were both normal. Computed tomo: $\frac{5}{5}$ raphy showed a small, low density, contrast enhancin lesion in the right posterior temporal lobe which extende to the lateral and posterior aspect of the temporal horn of the lateral ventricle (fig 2). Lumbar puncture and spingl fluid studies were normal. A tentative diagnosis of a low. 근 grade astrocytoma was made and the patient was begun $\overrightarrow{0}$ phenytoin. He was discharged to continue his trip home and advised to contact a physician for continuing care including another computed tomographic scan in three to six months.

Case 2. A 35-year-old woman was seen for neuroophthalmic evaluation because of a disturbance of vision on the left side. At the age of 22 years, she had the onset of $\frac{Q}{\Phi}$ headaches which were often preceded by a prodrome of $\varrho$ "squiggly lines" in the peripheral visual field and a scotoma $\overrightarrow{\overrightarrow{0}}$

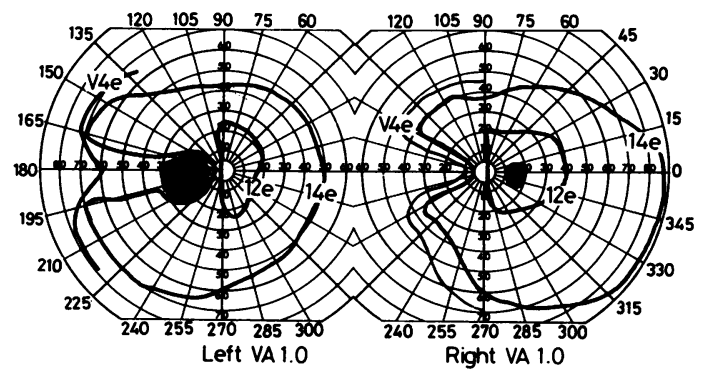

Fig 1 Visual fields of Patient 1 demonstrating a homonymous defect with sloping borders extending along the horizontal meridian to fixation. 
(a)
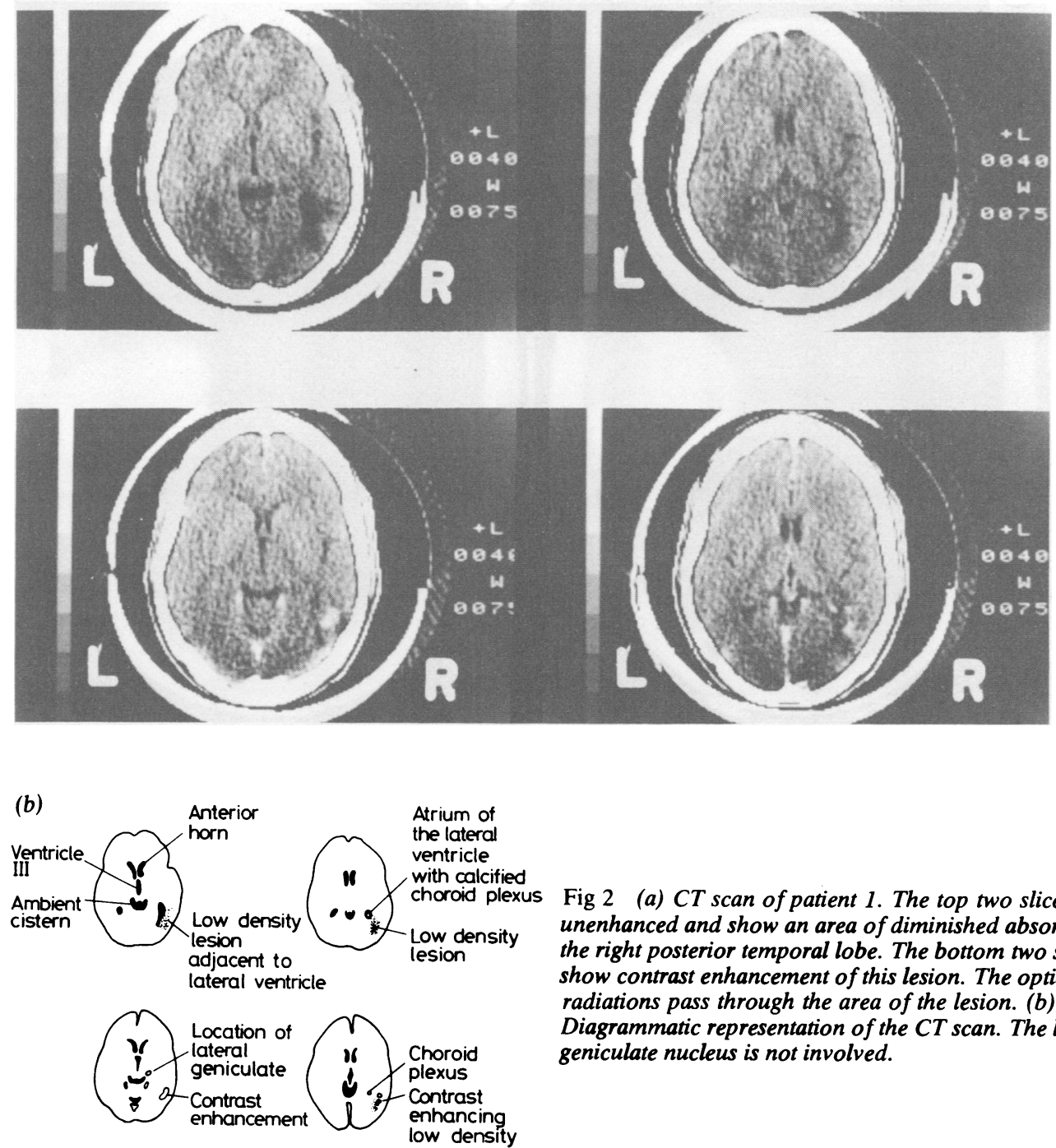

Fig 2 (a) CT scan of patient 1. The top two slices are unenhanced and show an area of diminished absorption in the right posterior temporal lobe. The bottom two slices show contrast enhancement of this lesion. The optic radiations pass through the area of the lesion. (b) Diagrammatic representation of the CT scan. The lateral geniculate nucleus is not involved.

in the central part of her visual field. The headaches were severe and were accompanied by nausea and vomiting. These were fairly well controlled by ergot preparations. She had a history of loss of consciousness associated with any episode of severe emesis since the age of ten years. These consisted of a period of light-headedness followed by loss of consciousness for less than one minute. During the period of unconsciousness, she sometimes experienced a few clonic movements of her extremities but had never bitten her tongue or been incontinent. These episodes of loss of consciousness occurred during the headaches only when there were nausea and vomiting but also were present at other times when vomiting occurred for other reasons. A neurological evaluation including skull series

and EEG had been normal six years after the onset of her headaches. One morning twelve years after the onset of her headaches, she experienced the violent onset of a severe headache associated with vomiting and became progressively less responsive during the next several hours. She was taken to a hospital where she was arousable but had a dilated and fixed pupil on the right side. She continued to deteriorate and exhibited decerebration in both upper extremities when stimulated. Babinski signs were present bilaterally. A computed tomographic scan showed a large intracerebral haematoma in the right parietotemporal region (fig 3). Right carotid arteriography showed a small arteriovenous malformation supplied by the posterior temporal branch of the right middle cerebral artery. 

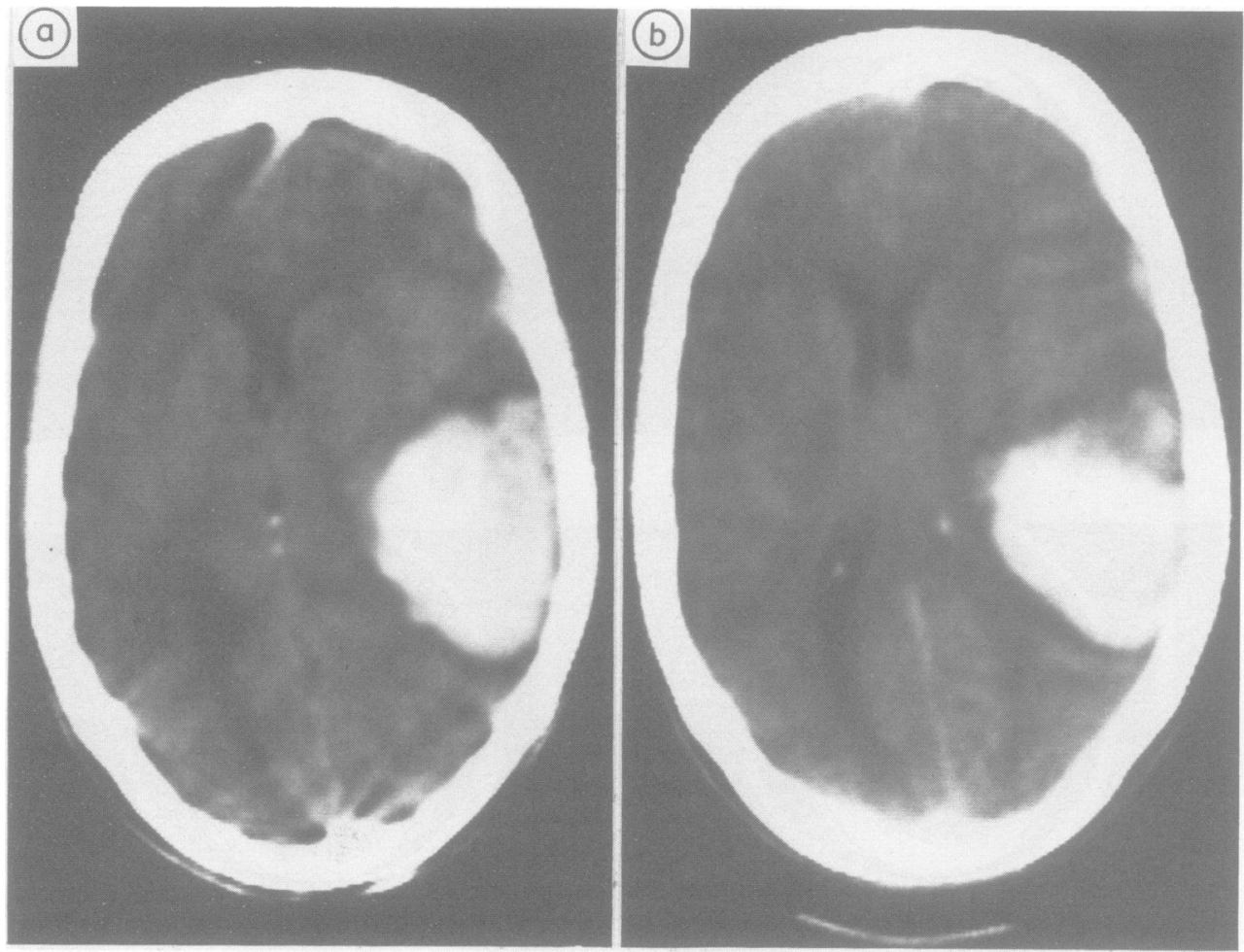

Fig 3 ( $a$ and $b$ ) CT scan of Patient 2 demonstrating a large intracerebral haematoma in the right parietotemporal region.

Active bleeding was seen during the arteriogram. The patient was taken to surgery where the haematoma was evacuated and the arteriovenous malformation was removed. A second craniotomy was performed to complete the removal of the arteriovenous malformation when a second arteriogram demonstrated persistent malformation. The patient did well postoperatively, recovering except for a mild left hemiparesis, subjective hemisensory disturbance, and visual field loss. Temporal lobe seizures and unformed visual hallucinations in the left visual field were controlled with phenytoin except for "swirly lines" occurring in the left visual field when she moved around in

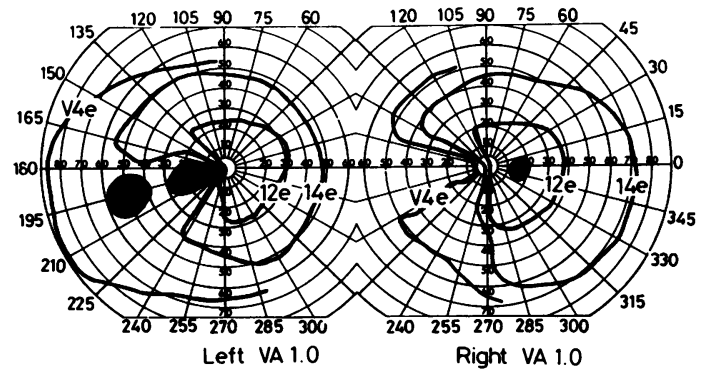

Fig 4 Visual fields of Patient 2 demonstrating a left homonymous horizontal sectoranopia. a dark room. Neuro-ophthalmic examination performed nine months after surgery was normal except for the pres? ence of a left homonymous horizontal sectoranopia (fig 4). Case 3 A 35-year-old male suffered severe head trauma resulting in a large intracerebral haematoma in the right temporal lobe. The haematoma was removed surgically and he was discharged with a residual left hemiparesis and a right Horner's syndrome. Confrontation visual fields suggested a left homonymous superior quadrantanopia but

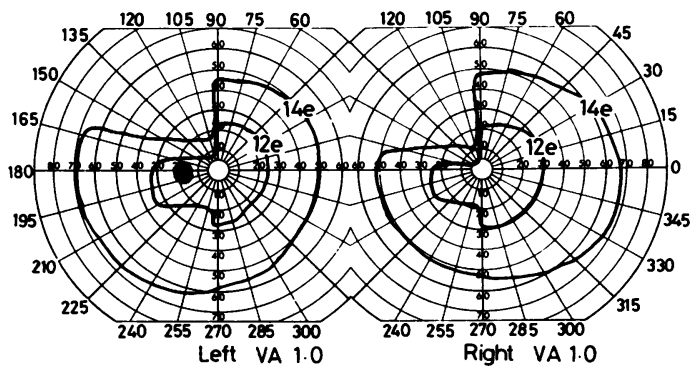

Fig 5 Visual fields of patient 3. A large homonymous superior quadrantanopia is accompanied by a smaller, relative, homonymous inferior quadrantanopia. The horizontal sector along the horizontal meridian is spared. 

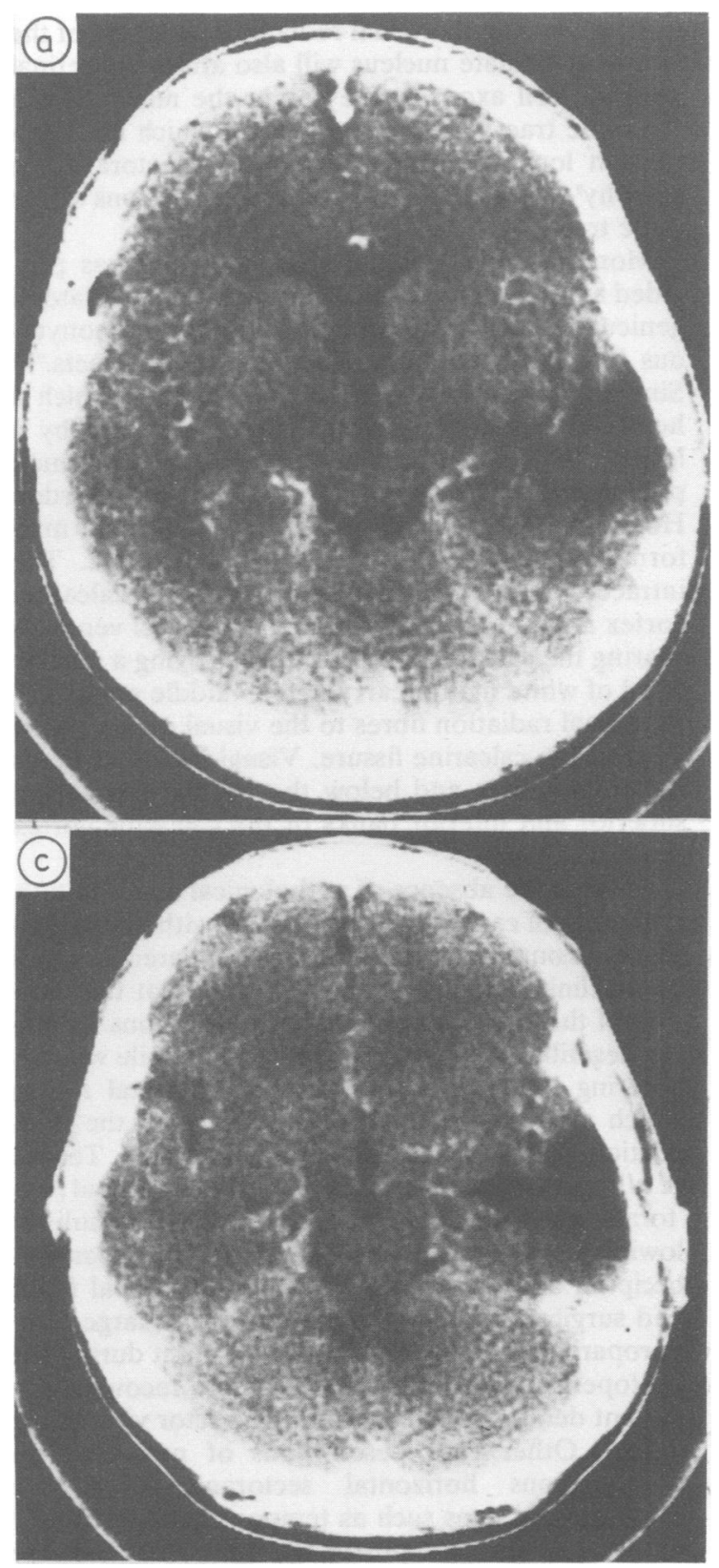

formal perimetry was not done. Following discharge, his family complained of a personality change consisting of emotional lability and poor attention span. For this reason, he was re-evaluated. Neuro-ophthalmic examination at that time showed a visual acuity of $6 / 12(20 / 40)$ in the right eye and $6 / 6(20 / 20)$ in the left eye. The right pupil was slightly smaller than the left and cocaine and peredrine testing confirmed the presence of preganglionic Horner's (b)

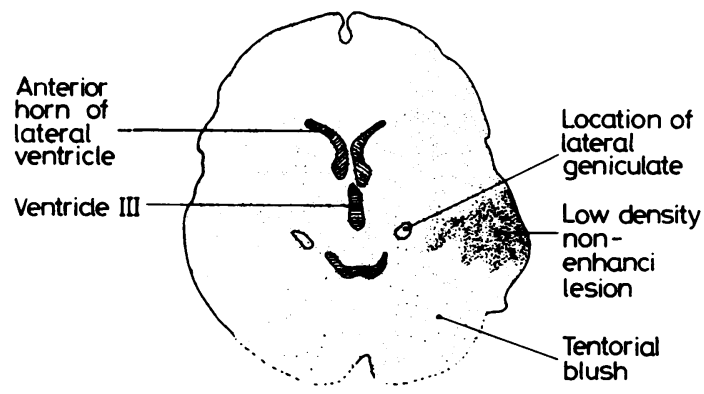

(d)

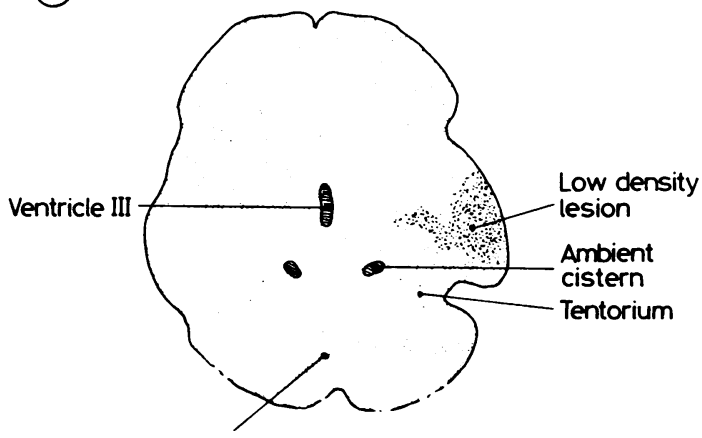

Cerebellum

Fig $6 C T$ scan and diagrammatic representation of the fundings of Patient 3 at the time of visual field testing. The intracerebral haematoma has resolved leaving an area of low absorption which is located in the right temporal lobe. $(A$ and $B)$ The region of the lateral geniculate nucleus on the right side is identical to its counterpart on the left side. The low density lesion on the right side is within the temporal lobe, its deep extension being behind the tentorial blush. (C and D) At a level slightly below $A$. and $B$. and below the level of the lateral geniculate nucleus, the low density lesion extends into the deep white matter of the temporal lobe where the optic radiation fibres are located.

syndrome. The right eye also had a mild relative afferent pupillary defect. Visual fields demonstrated a left homonymous "quadruple sectoranopia"10 (fig 5). Fundus examination showed mild temporal pallor of the right disc, but the left optic disc and peripapillary nerve fibre layer were normal. Computed tomography showed a lucency at the site of the old intracerebral haematoma in the right temporal lobe. The damaged area extended deep into the 
substance of the temporal lobe, but deep extension of the lesion was behind the ambient cistern and did not involve the region of the lateral geniculate nucleus (fig 6). The visual field defect was felt to be a residual deficit from the temporal lobe lesion. The other findings were felt to be due to mild traumatic optic neuropathy. While a right optic tract/lateral geniculate lesion might have produced the visual field defect, this was not a plausible explanation for two reasons. First, the optic atrophy was limited to the right eye rather than being the characteristic sectoral optic atrophy seen in both eyes of patients with lesions in the lateral geniculate nucleus or optic tract. ${ }^{811}$ Second, relative afferent pupillary reaction defects may be seen with optic tract lesions but are usually present only when the homonymous hemianopia is complete ${ }^{1213}$ and are found in the contralateral eye..$^{12-14}$

\section{Discussion}

The anatomical and physiological features of the lateral geniculate nucleus and its blood supply which allow the occurrence of homonymous horizontal sectoranopias have been discussed by several authors. $^{7-91015}$ Selective involvement of the larger middle section of the lateral geniculate nucleus produces the more common homonymous defect in which a single sector extends from the periphery along the horizontal meridian to fixation..$^{7-9} 15$ Less commonly, involvement of the lateral and medial horns of the lateral geniculate nucleus spare the segment of the visual field along the horizontal meridian but produce homonymous visual field defects above and below this segment, producing what Frisen labelled a "quadruple sectoranopia". ${ }^{10}$ For reasons discussed by Hoyt ${ }^{8}$ incongruity may be such that only three of the possible four sectors are affected.

There has been only one case in which a homonymous horizontal sectoranopia was attributed to a lateral geniculate nucleus lesion and in which pathologic material was subsequently available. ' Unfortunately, the visual field defect had progressed to a complete homonymous hemianopia and a tumour involved not only the lateral geniculate nucleus but also the mesial temporal lobe, thalamus and subthalamus, basal ganglia and the midbrain and pontine tegmentum. The lateral geniculate nucleus appeared close to the geometric centre of this extensive tumour, but a partial complex seizure disorder had been present for 19 years prior to any visual symptoms, suggesting that the temporal lobe could as well have been the initial site of involvement by the tumour.

Despite the absence of pathological studies, certain clinical features and neuroradiological data may allow localisation of a lesion causing a homonymous horizontal sectoranopia to the lateral geniculate nuc- leus rather than the visual radiations. A lesion of the lateral geniculate nucleus will also affect the retinal ganglion cell axons as they enter the nucleus from the optic tract. Therefore, a lesion which has been present long enough will produce a sectoral optic atrophy $y^{8-10}$ identical to that seen with lesions of the optic tract. ${ }^{11}$

More recently, computed tomography has provided anatomical data localising lesions to the lateral geniculate nucleus in three patients with homonymous horizontal sectoranopia visual field defects. ${ }^{9}$ is Similarly, there has been only one case in which a homonymous horizontal sectoranopic caused by a lesion of the optic radiations was confirmed pathologically. This was presented by Gordon Holmes in $1931^{3}$ and involved an arteriovenous malformation and an intracerebral haematoma. The intracerebral haematoma lay between the calcarine cortex and the occipital horn of the lateral ventricle sparing the calcarine cortex but destroying a narrow band of white matter carrying the middle portion of the visual radiation fibres to the visual cortex in the floor of the calcarine fissure. Visual radiation fibres travelling above and below the haematoma to the superior and inferior banks of the calcarine cortex remained intact.

Despite the absence of pathological confirmation $\frac{}{10}$ a number of case reports of patients with homonym $\%$ ous horizontal sectoranopia have appeared in whick 0 other clinical details strongly suggest that the loca 80 tion of the lesion was in the optic radiations. Spaldo ing described four such patients with missile wounds entering the skull in the temporo-parietal regio which were believed to have penetrated the midportion of the visual radiation. ${ }^{4}$ Similarly, Teuber et al $^{16}$ described one patient with horizontal sectoranopic visual field defects to coloured stimuli following a missile injury to the contralateral parietooccipital area. Traquair illustrates the visual fields and surgical findings in a patient with a large temporoparietal meningioma. ${ }^{17}$ At one point during the postoperative evolution of visual field recovery, the patient demonstrated a quadruple sector visual field defect. Other case descriptions of patients with homonymous horizontal sectoranopias include symptoms or signs such as temporal lobe seizures ${ }^{56}$ or visual agnosia and prosopagnosia ${ }^{18}$ which indicate a lesion in the temporal lobe or temporo-parietal region rather than in the lateral geniculate nucleus. Figure 7 indicates the proposed placement of the lesion in the visual radiations adjacent to the atrium of the lateral ventricle and the corresponding visual field defect seen in Patient 1 of this report. In all three patients presented here, computed tomography provides anatomical evidence that homonymous horizontal sectoranopias were the result 


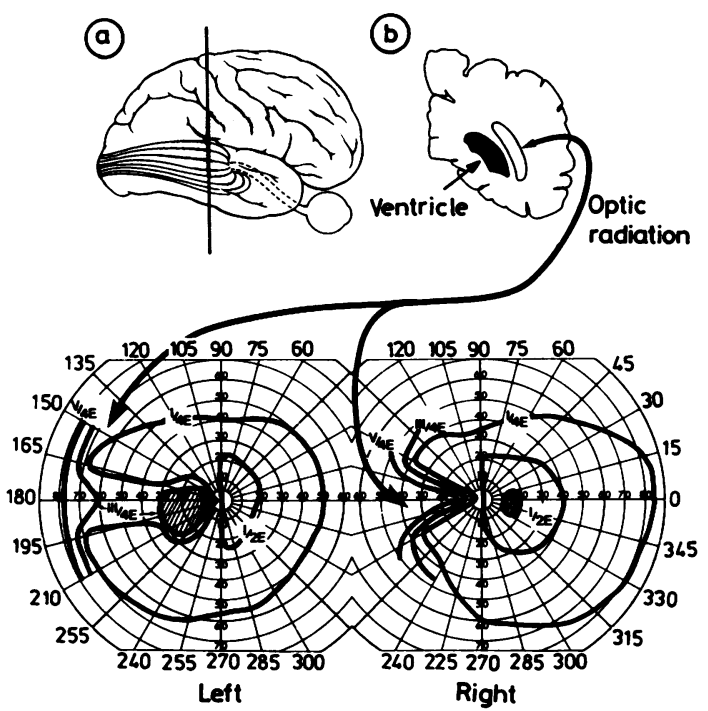

Fig 7 Visual fields of patient 1 and diagrammatic representation of the visual radiations seen from the side $(A)$ and in cross-section (B). The shaded area is the proposed site of the lesion for Patients 1 and 2. The site of damage in Patient 3 would include the inferior unshaded area producing the superior quadrantanopia and less extensive involvement of the superior unshaded area producing the mild inferior quanrantanopia.

of lesions of the visual radiations in the temporoparietal lobe rather than lesions of the lateral geniculate nucleus itself.

It appears evident from the present cases that homonymous horizontal sectoranopic visual field defects can be the result of lesions either in the lateral geniculate nucleus or in the optic radiations. Additional clinical data available at the time of the patient's evaluation are likely to localise the lesion. A history of disturbance in visual memory integration such as visual agnosia or prosopagnosia or a history of seizures argues strongly for a lesion of the visual radiations in the cerebral hemispheres. If there is a history of brief, episodic behavioural disturbances, the possibility that these represent partial complex seizures should arise and further neurological evaluation should be considered. The presence of sectoral optic atrophy provides firm evidence of a lesion of the lateral geniculate nucleus. A combination of these signs is possible and would indicate an extensive lesion, probably neoplastic, involving both the lateral geniculate nucleus and the cerebral hemispheres as seen in Gunderson and Hoyt's patient.' If history, examination and contrast enhanced computed tomography all fail to demonstrate a lesion site, arteriography should be consi- dered. Although homonymous horizontal sectoranopic visual field defects are uncommon, three of the reported cases have had small arteriovenous malformations in the ambient cistern, ${ }^{79}$ 15 one had an aneurysm of the internal carotid artery, ${ }^{18}$ and case two of this report had a small arteriovenous malformation supplied by a temporal branch of the middle cerebral artery.

This study was supported in part by an unrestricted development grant from Research to Prevent Blindness.

\section{References}

I Smith JL. Homonymous hemianopia: a review of one hundred cases. Am J Ophthalmol 1962;54:616-22.

${ }^{2}$ Trobe JD, Lorbes ML, Schleizinger NS: Isolated homonymous hemianopia. Arch Ophthalmol 1973;89:377-81.

${ }^{3}$ Holmes G. A contribution to the cortical representation of vision. Brain 1931;54:470-9.

${ }^{4}$ Spalding JMK. Wounds of the visual pathway. Part I: The visual radiation. J Neurol Neurosurg Psychiatry 1952; 15:99-107.

s Smith RJS. Horizontal sector hemianopia of nontraumatic origin. $\mathrm{Br} J$ Ophthalmol 1970;54:208-10.

${ }^{6}$ Fite JD. Temporal lobe epilepsy. Association with homonymous hemianopia. Arch Ophthalmol 1967;77:71-75.

${ }^{7}$ Gunderson $\mathbf{C H}$, Hoyt WF. Geniculate hemianopia: incongruous homonymous field defects in two patients with partial lesions of the lateral geniculate nucleus. J Neurol Neurosurg Psychiatry 1971;34:1-6.

${ }^{8}$ Hoyt WF. Geniculate hemianopias: incongruous visual defects from partial involvement of the lateral geniculate nucleus. Proc Aust Assoc Neurol 1975;12:19-28.

${ }^{9}$ Frisen L, Holmegaard L, Rosencranz M. Sectorial optic atrophy and homonymos, horizontal sectoranopia: a lateral choroidal artery syndrome? J Neurol Neurosurg Psychiatry 1978;41:374-80.

${ }^{10}$ Frisen L. Quadruple sectoranopia and sectorial optic atrophy: a syndrome of the distal anterior choroidal artery.J Neurol Neurosurg Psychiatry 1979;42:590-4.

"Hoyt WF, Kommerell G. Der fundus oculi bei homonymous hemianopie. Klen Monatsbl Augenheilkd 1973;162:456-64.

12 Bell RA, Thompson HS. Relative afferent pupillary defect in optic tract hemianopias. Am J Ophthalmol 1978;85:538-40.

${ }^{13}$ Newman SA, Miller NR. Optic tract syndrome. Neuroopthalmologic considerations. Arch Opthalmol 1983; 101:1241-50.

${ }^{14}$ O'Connor PS, Kasdon D, Tredici TJ, Ivan DJ. The Marcus-Gunn pupil in experimental optic tract lesions. Ophthalmology 1982;89:160-4.

is Shacklett DE, O'Connor PS, Dorwart RH, Linn D, Carter JE. Congruous and incongruous sectoral visual field defects with lesions of the lateral geniculate nucleus. Am J Ophthalmol 1984;98:283-90. 
16 Teuber HL, Battersby WS, Bender MB. Visual Field Defects after Penetrating Missile Wounds of the Brain. Cambridge, Harvard University Press, 1960:73.

${ }^{17}$ Scott GI. Traquair's Clinical Perimetry. London, Henry Kimpton, 1975:269-70.

${ }^{18}$ Oosterhuis HJGH, Ponsen L, Jonkman EJ, Mangus $O$.
The average visual response in patients with cerebrovascular disease. Electroencephalogr Clin Neurophysiol 1969;27:23-34.

${ }^{19}$ Kearns TP, Rucker CW. Clinics in perimetry no. 3: Incongruous homonymous hemianopia. $\mathrm{Am} J$ Ophthalmol 1959;47:317-21. 